\title{
Up-Wave and Autoregressive Methods for Short-Term Wave Forecasting for an Oscillating Water Column
}

\author{
Francesco Paparella, Kieran Monk, Victor Winands, M. F. P. Lopes, Daniel Conley, and John V. Ringwood
}

\begin{abstract}
The real-time control of wave energy converters (WECs) requires the prediction of the wave elevation at the location of the device in order to maximize the power extracted from the waves. One possibility is to predict the future wave elevation by combining its past history with the spatial information coming from a sensor which measures the free surface elevation up-wave of the WEC. As an application example, this paper focuses on the prediction of the wave elevation inside the chamber of the oscillating water column (OWC) for the Pico OWC plant in the Azores, and two different sensors for the measurement of the free surface elevation up-wave of the OWC were tested. The study showed that the use of the additional information coming from the up-wave sensor does not significantly improve the linear prediction of the chamber wave elevation given by a forecasting model based only on the past values of the chamber wave elevation.
\end{abstract}

Index Terms-Time series, wave energy, wave forecasting.

\section{INTRODUCTION}

W AVE ENERGY converters (WECs) are usually designed to efficiently extract energy from the sea for a limited range of frequencies, usually located around the resonance of the device. In order to realize an efficient energy extraction for a broader range of frequencies, a control system can be designed [1], [2]. Real-time optimal control can be derived from a controller designed in the frequency domain, but it involves noncausal transfer functions which can be implemented only if the future motion of the device or the incident wave elevation are known [1], [2]. Usually, prediction of the wave elevation is based on a spatial reconstruction of the wave field starting from a set of sensors located in the proximity of

Manuscript received June 04, 2014; revised August 01, 2014; accepted September 18, 2014. Date of publication December 02, 2014; date of current version December 12, 2014. Paper no. TSTE-00268-2014.

F. Paparella and J. V. Ringwood are with the Center for Ocean Energy Research (COER), National University of Ireland, Maynooth, Ireland (e-mail: fpaparella@eeng.nuim.ie; john.ringwood@eeng.nuim.ie).

$\mathrm{K}$. Monk is with the School of Marine Science and Engineering, University of Plymouth, Plymouth PL48AA, U.K., and also with the WAVEC Offshore Renewables, Lisbon 1400-119, Portugal (e-mail: Kieran.Monk@plymouth. ac.uk).

V. Winands is with Duespan Wave Engineering, 14167 Berlin, Germany (e-mail: Duespan@googlemail.com).

M. F. P. Lopes are with the WAVEC Offshore Renewables, Lisbon 1400-119, Portugal (e-mail: mlopes@wavec.org).

D. Conley is with the School of Marine Science and Engineering, University of Plymouth, Plymouth PL48AA, U.K. (e-mail: daniel.conley@plymouth. ac.uk).

Color versions of one or more of the figures in this paper are available online at http://ieeexplore.ieee.org.

Digital Object Identifier 10.1109/TSTE.2014.2360751 the WEC, as shown in Fig. 1(b) [3]. Another possible solution is to predict the wave elevation based only on its past history, as shown in Fig. 1(a). The prediction of wave elevation based on past values requires less instrumentation respect to the spatial approach, since only one measure is needed at the sea location of the WEC. Regarding spatial approach for wave prediction in [4], digital filters were deployed for the real-time prediction of waves incident upon a wave energy device. In [5], the remote wave profile is measured by means of a nautical radar and is propagated in time and space in order to predict the wave field and estimate the motion of a vessel. In [6], a shallow angle LIDAR was adopted in order to measure the time evolution of spatial profiles of sea waves over an extended region of several hundred meters. In [7], the fast Fourier transform is employed to decompose the wave elevation into individual frequencies at a given point, propagate each individual frequency component using the dispersion relation, and reconstruct the wave elevation at a different temporal and spatial point. In [8], an overview of a deterministic sea-wave prediction (DSWP) model is presented, which is used to estimate the wave elevation at the point of interest, given the measurement of the free surface elevation at a point at some distance from the prediction site. In [9], a linear harmonic model fitted to distant wave elevation measurements is presented. In [10], the hydrodynamics of the chamber of an oscillating water column (OWC) is predicted by means of a neural network (NN) based on the measurements of the incident wave elevation.

Regarding time-series approach for wave prediction, in [3] autoregressive (AR) models are proposed and validated against real observations. In [11], adaptive filters based on AR models for wave prediction are deployed. In [12], a robust control for wave energy devices based on the prediction of the wave elevation is implemented. For the wave prediction, a hybrid Kautz/AR predictive model as well as a purely predictive Kautz model are proposed. In [13], NNs for the estimation of the wave excitation force were trained and results compared to other methods.

This paper examines the comparative benefits on wave prediction accuracy using a sensor for the measurement of the free surface elevation up-wave of the WEC, compared to using historical measurements alone. In particular, an AutoRegressive eXogenous (ARX) input model, which extends the AR model with the inclusion of the additional information coming from the measurement of the free surface elevation up-wave of the WEC. The up-wave elevation is considered to be an exogenous input of the model. The ARX models proposed are 


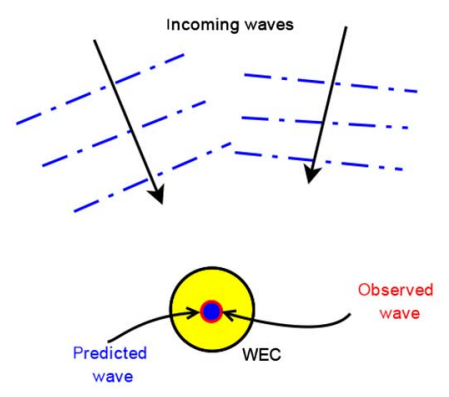

(a)

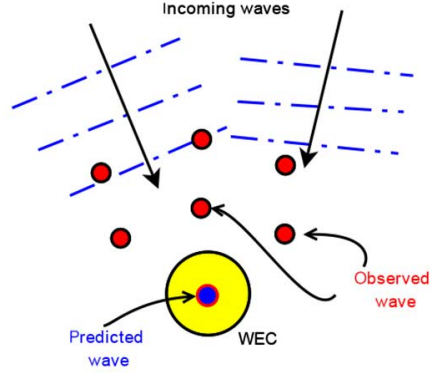

(b)
Fig. 1. (a) Wave prediction based on one-single point of measurements and (b) wave prediction based on spatial reconstruction from array of sensors

validated against real observations coming from the Pico WEC plant located on the Portuguese island of Pico in the Azores archipelago [10]. In particular, two types of sensors for the upwave elevation have been tested. The real data available are discussed in Section II, while the forecasting models proposed are outlined in Section III. Finally, the results are discussed in Section IV.

\section{AVAilable DATA}

This paper proposes a strategy for forecasting the wave elevation inside the chamber of the Pico OWC by a combination of its past values and the measurement of the free surface elevation up-wave of the WEC. A sonar sensor for recording the chamber wave elevation is mounted on the roof of the chamber, while two different sensors for the measurement of the free surface elevation around $60 \mathrm{~m}$ in front of the Pico OWC chamber wall are deployed [10]. During the first deployment period, the "Aquadopp" hydrostatic pressure sensor and Acoustic Doppler Current Meter (ADCM) unit was utilized to measure the hydrodynamics of the free surface elevation up-wave of the WEC. Since the data cable providing the online data from the sensor was damaged by the sea, the sensor was redeployed from time to time in order to acquire the data stored in the internal memory of the device. Obviously, for the wave elevation forecasting inside the chamber, an online measurement of the free surface up-wave elevation is needed. Given the high cost for the data cable, an alternative sensor was developed and used at the same location as the Aquadopp during the second deployment period. This alternative sensor, in the following referred with the name "pneumatic sensor," consists of a small steel box fixed on the sea floor with an open bottom. The variation of the pressure of the entrapped air inside the box, due the change in the water column above, is measured by a pressure transducer. From the pressure variation of the air inside the box, the water surface elevation can be obtained [10] as

$$
\eta_{w}=\frac{p_{\text {hyd }}}{\rho g}
$$

where $p_{\text {hyd }}$ represents the dynamic water pressure at $60 \mathrm{~m}$ in front of the OWC, $\rho$ is the density of the sea water, and $g$ is the gravitational acceleration. The data available from the Aquadopp sensor consist of $25 \mathrm{~h}$ of records, while the data provided by the pneumatic sensor consist of $10 \mathrm{~h}$ of records.

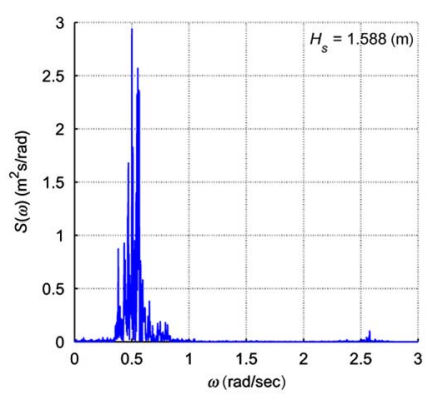

(a)

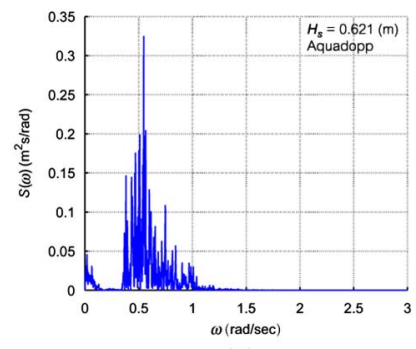

(c)

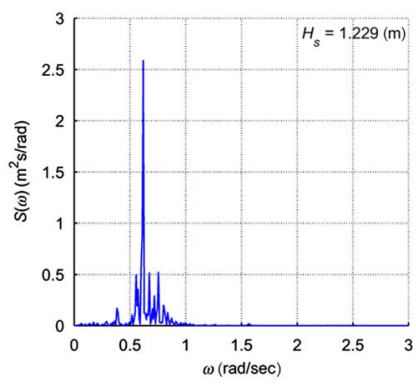

(b)

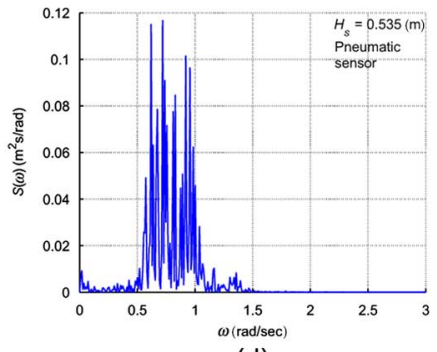

(d)
Fig. 2. Chamber wave elevation spectra for (a) first and (b) second deployment period and up-wave elevation spectra from (c) Aquadopp sensor and from (d) pneumatic sensor.

The sampling frequency for both the deployment periods is equal to $2 \mathrm{~Hz}$.

The spectra of the chamber wave elevation measured during the first and second deployment period are reported in Fig. 2(a) and (b), respectively, while the corresponding spectra of the up-wave elevation measured with the Aquadopp and pneumatic sensor are reported, respectively, in Fig. 2(c) and (c). The spectra of the chamber wave elevation show a resonance around $0.62 \mathrm{rad} / \mathrm{s}$ for both deployment periods, which corresponds to a dominant wave period of approximately $10 \mathrm{~s}$. However, the spectra of the free surface elevation up-wave of the WEC show that, in addition to the dominant wave frequency, other components exist at higher frequency. Because the incident waves are reflected by the wall of the OWC, frequency components of shorter period can be present in the spectra of up-wave elevation [10].

Since the low-frequency components carry most of the wave energy, it is a reasonable assumption to predict only the lowfrequency components of the chamber wave elevation [3]. In fact, the high frequencies are representative of low energy sea states, which are not relevant for wave energy conversion. Also, neglecting the sea spectra components at high frequency yields to a more regular and more predictable wave elevation, and accurate predictions can be also obtained for long forecasting horizons. Therefore, the data are filtered with an ideal zero-phase low-pass filter with cut-off frequency $\omega_{c}$. The filter is approximated with offline forward and backward filtering through Chebyshev filters of Type I, discretized with the bilinear transform. The cut-off frequency was selected in order to preserve the dominant wave period of the sea state.

If the free surface elevation up-wave of the WEC propagates only in the direction toward the chamber, with a time delay termed the propagation time, the incident wave elevation causes the change in the chamber wave elevation. 
The propagation time can be inferred from considerations of wave propagation in a finite water depth. Assuming that there are no attenuation effects on the traveling wave, the wave elevation $\eta$ propagates in the direction $x$, defined positive from the location of the up-wave sensor to the chamber, accordingly to the following expression [14]:

$$
\eta(t, x)=A \cos (\omega t-k(\omega) x)=\operatorname{Re}\left(A e^{j(\omega t-k(\omega) x)}\right)
$$

where $k(\omega)$ is the wave number, which is a function of the frequency $\omega$ and water depth $h$. The wave propagates with a phase velocity given by [2]

$$
v_{p}=\frac{\omega}{k(\omega)} .
$$

In shallow water with depth $h$, the waves can reach a maximum velocity $v_{\max }=\sqrt{g h}$, where $g$ is the gravitation acceleration. Since the up-wave sensor is placed at a location with a water depth $h=8 \mathrm{~m}$, a velocity $v_{\max }=8.85 \mathrm{~m} / \mathrm{s}$ is obtained. If it is assumed that all the waves travel at the same phase velocity equal to the maximum velocity, then the free surface elevation up-wave of the WEC reaches the chamber without any phase distortion. If $d$ denotes the spatial distance between the point of measurement of the free surface elevation up-wave of the WEC and the point of measurement of the chamber elevation, the propagation time $t_{\text {prop }}$ of the wave is as follows [14]:

$$
t_{\text {prop }}=\frac{d}{v_{\max }} .
$$

Since the point of measurement of the up-wave elevation is placed at a distance $d=60 \mathrm{~m}$ from the chamber, using (4), a propagation time around $7 \mathrm{~s}$ is obtained. If there are no nonlinearities or attenuation effects on the wave propagation, the use of the up-wave elevation measurement provides advance knowledge of the future wave elevation inside the chamber for a forecasting horizon equal to the propagation time. In reality, the presence of eventual nonlinearities on the wave propagation and multidirectionality of the waves distorts the effect of the incoming wave on the chamber wave elevation. As a result, the measurement from the up-wave sensor gives a partial information about the future wave elevation inside the chamber. As shown in (4), the distance of the up-wave sensor from the chamber determines the maximum forecasting horizon. The measurements of the up-wave elevation given by a sensor located at a long distance from the chamber provides less accurate information about the future chamber wave elevation, due to the increasing effect of nonlinearities and multidirectional waves. Also, in order to acquire the measurements from a sensor more distant from the site where the prediction is required, greater costs for the data cable are involved.

\section{FORECASTING MODELS}

\section{A. Up-Wave Only Models}

In this section, a model for forecasting the chamber wave elevation based only on the measurement of the free surface

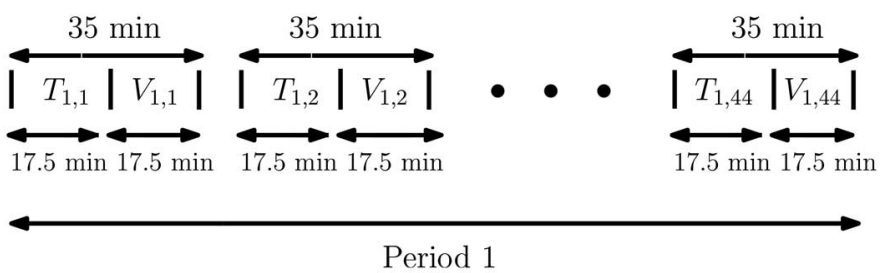

(a)

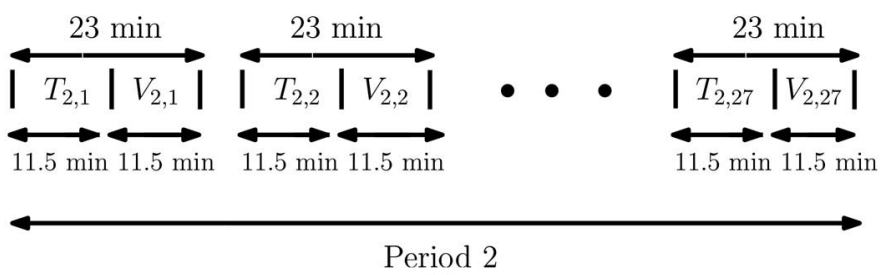

(b)

Fig. 3. Time-series data segmentation for the (a) first and (b) second deployment period.

elevation up-wave of the WEC is presented. In particular, a finite impulse response (FIR) model is designed which assumes that the chamber wave elevation at time instant $k$ is a linear combination of $n_{b}$ past values of the free surface elevation upwave of the WEC. The up-wave measurement is considered to be the input of the model and is denoted as $u$. The model considered is of the following form:

$$
\eta_{\mathrm{ch}}(k)=\sum_{i=1}^{n_{b}} b_{i} u\left(k-i+1-n_{k}\right)+\xi(k)
$$

where $\xi$ is considered to be a white noise with zero mean and variance $\sigma^{2}$. The term $n_{k}$ is the delay (in number of sample periods) that occurs before the output is affected by the input. If the data coming from the up-wave sensor are delayed by the propagation time then, at time instant $k$, the water level in the chamber is influenced by the up-wave elevation at the same time instant. Therefore, the delay $n_{k}$ is considered to be zero. The use of the FIR filter takes into account the unmodeled dynamics between the up-wave elevation and the chamber wave elevation which was not considered in the calculation of the propagation time alone. Also, the FIR filter models the dynamics of the sensor utilized for the measurement of free surface elevation up-wave of the WEC. In fact, since the up-wave sensor deployed approximates the free surface elevation based on a pressure signal, the FIR filter also models the dynamic relationship between the pressure and the free surface elevation. Given a set of parameters $b_{i}$, from (5), the $l$-step ahead prediction is given as follows:

$$
\hat{\eta}_{\mathrm{ch}}(k+l \mid k)=\sum_{i=1}^{n_{b}} b_{i} u(k+l-i+1) .
$$

In (6), the time series of the up-wave elevation is known up to a certain prediction horizon, equal to the propagation time of the wave from the location of the sensor.

As shown in Fig. 3(a) and (b), the data for each deployment period were divided into training and validation sets. The coefficients $b_{i}$ of the FIR model, at an instant $k$, are estimated using 
a batch of training data $T_{i, j}$ of dimension $N_{1}$ consisting of past observations of the water level in the chamber and measurements of the free surface elevation up-wave of the WEC. The cost function to be minimized, for the estimation of the model parameters, is the variance of the one-step ahead prediction error, which is given as follows:

$$
J_{\mathrm{LS}}=\sum_{k=1}^{N_{1}}\left(\eta_{\mathrm{ch}}(k+1)-\hat{\eta}_{\mathrm{ch}}(k+1 \mid k)\right)^{2}
$$

which is a linear least squares (LS) problem. For the estimation of the model parameters, a different cost function referred as long range predictive identification (LPRI) [15] was considered, with initial value of the parameters given by the minimization of (7). Since the minimization of the LPRI cost function for different forecasting horizons did not yield a significantly different value of the parameters compared to their initial estimates, the LPRI cost function was not further considered.

An important element of the identification of the FIR model with cost function (7) is the choice of the order $n_{b}$. The objective is to identify a model that guarantees an accurate fitting of the training data without incurring the so-called "over-fitting," or rather the identification of a over-parametrized model which also interpolates the stochastic noise presents in the measurements. Given a previously unseen batch of validation data $V_{i, j}$ of dimension $N_{2}$ consisting of observations of the chamber and up-wave elevations, the accuracy of the identified model can be validated by means of the variance of the prediction error $\sigma_{l}^{2}$ for a forecasting horizon $l$ computed as follows:

$$
\begin{aligned}
\sigma_{l}^{2} & =\frac{1}{N_{2}} \sum_{k=1}^{N_{2}} \hat{e}(k+l \mid k)^{2} \\
& =\frac{1}{N_{2}} \sum_{k=1}^{N_{2}}\left(\eta_{\mathrm{ch}}(k+l)-\hat{\eta}_{\mathrm{ch}}(k+l \mid k)\right)^{2} .
\end{aligned}
$$

In order to select the best order $\bar{n}_{b}$ that avoids over-fitting the data, FIR filters of different orders $n_{b}$ were trained and validated by means of the data coming from the two deployment periods. In Fig. 4, the variance $\sigma_{l}^{2}$ for a forecasting horizon equal to the propagation time of $7 \mathrm{~s}$ is computed for FIR filters of different order $n_{b}$. The variance is averaged across the validation data sets coming from both the deployment periods, normalized by the appropriate maximum value. An order $n_{b}$ equal to 10 is chosen as point of trade-off between the minima of the two curves.

\section{B. AR Models}

In this section, a model for forecasting the chamber wave elevation, based on its past history, is presented. In particular, an AR model is proposed which assumes that the chamber wave elevation at time instant $k$ is linearly dependent on a number $n_{a}$ of its past values. Thus, the model considered is of the following form:

$$
\eta_{\mathrm{ch}}(k)=\sum_{i=1}^{n_{a}} \alpha_{i} \eta_{\mathrm{ch}}(k-i)+\xi(k) .
$$

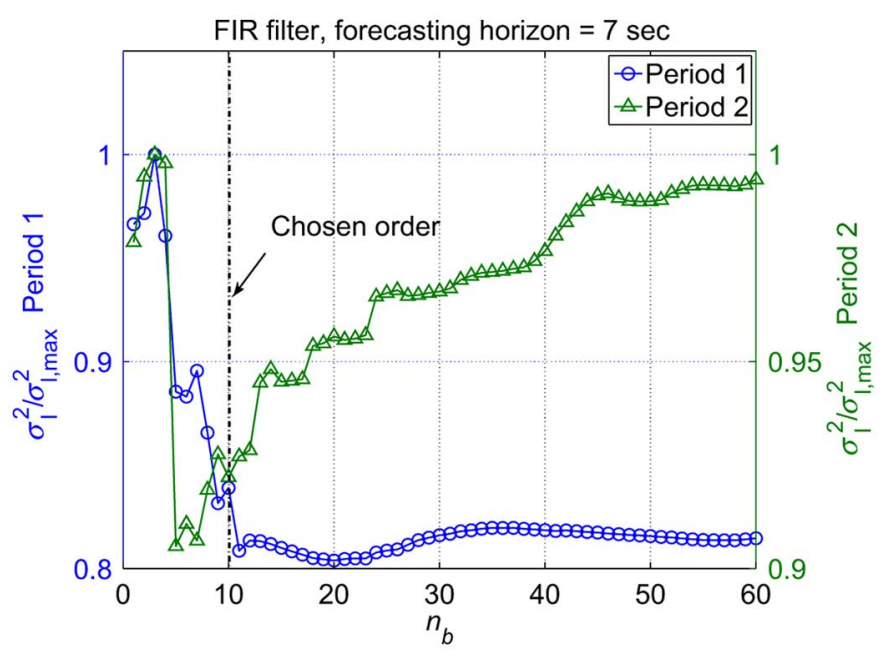

Fig. 4. Normalized prediction error variance for a forecasting horizon of $7 \mathrm{~s}$ as a function of the order $n_{b}$ averaged across validation data sets for the first and second deployment periods.

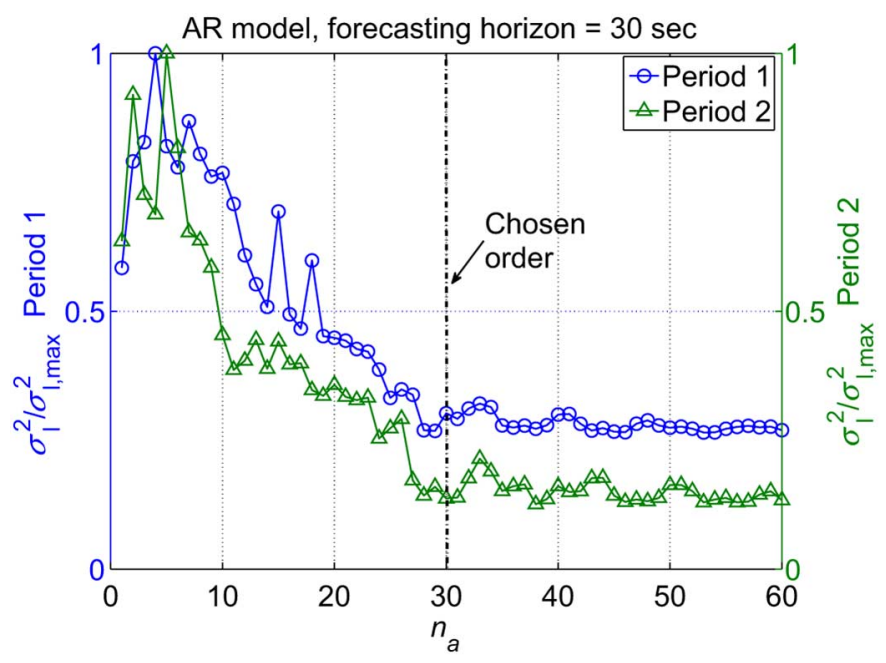

Fig. 5. Normalized prediction error variance for a forecasting horizon of $30 \mathrm{~s}$ as a function of the order $n_{a}$ averaged across validation data sets for the first and second deployment periods.

Given a set of parameters $\alpha_{i}$, from (9) the $l$-step ahead prediction is given as follows:

$$
\hat{\eta}_{\mathrm{ch}}(k+l \mid k)=\sum_{i=1}^{n_{a}} \alpha_{i} \hat{\eta}_{\mathrm{ch}}(k+l-i \mid k)
$$

where $\hat{\eta}_{\mathrm{ch}}(k+l-i \mid k) \equiv \eta_{\mathrm{ch}}(k+l-i) \quad$ if $\quad k+l-i \leq k$, since the information is already available and there is no need to have a prediction [3]. The coefficients $\alpha_{i}$ of the AR model are estimated using the cost function given by (7), and the identified models can be validated according to the variance of the prediction error over a forecasting horizon $l$, given by (8). AR models of different orders $n_{a}$ were trained and validated by means of the measurements of the wave elevation inside the chamber for both the deployment periods. In Fig. 5, the variance $\sigma_{l}^{2}$ for a forecasting horizon equal to $30 \mathrm{~s}$, normalized by its maximum value and averaged across the validation sets, is plotted against different order $n_{a}$ for both the deployment periods. An order 
$n_{a}$ equal to 30 is chosen since it represents a minimum point for both the two curves.

\section{Combination Up-Wave/AR Models}

In this section, a model for forecasting the chamber wave elevation based on a combination of its past history and measurements of the free surface elevation up-wave of the WEC is presented. In particular, an ARX input model is proposed which assumes that the chamber wave elevation at time instant $k$ is linearly dependent on a number $n_{a}$ of its past values and on $n_{b}$ values of the up-wave measure. Thus, the model considered is of the following form:

$$
\eta_{\mathrm{ch}}(k)=\sum_{i=1}^{n_{a}} a_{i} \eta_{\mathrm{ch}}(k-i)+\sum_{i=1}^{n_{b}} b_{i} u(k-i+1)+\xi(k) .
$$

Given a set of parameters $a_{i}$ and $b_{i}$, from (11), the $l$-step ahead prediction is given as follows:

$$
\begin{aligned}
\hat{\eta}_{\mathrm{ch}}(k+l \mid k)= & \sum_{i=1}^{n_{a}} a_{i} \hat{\eta}_{\mathrm{ch}}(k+l-i \mid k) \\
& +\sum_{i=1}^{n_{b}} b_{i} u(k+l-i+1) .
\end{aligned}
$$

In (12), no prediction of the input is made until a prediction horizon, equal to the propagation time of the wave from the location of the sensor is reached. The limit of the prediction horizon, in time steps, is denoted as $l_{\max }$, which is given as follows:

$$
l_{\text {max }}=\frac{t_{\text {prop }}}{t_{\mathrm{samp}}}=\frac{d / v_{p}}{t_{\mathrm{samp}}}
$$

where $t_{\text {samp }}$ is the sampling time of the chamber and up-wave elevation. Therefore, the prediction of $\hat{\eta}_{\text {ch }}$ can be made with an ARX model only until $l_{\max }$ steps, after which an AR only model is used to predict the water level in the chamber. Thus, the complete prediction of the wave elevation using an ARX model is given as follows:

$$
\hat{\eta}_{\mathrm{ch}}(k+l \mid k)= \begin{cases}\sum_{i=1}^{n_{a}} a_{i} \hat{\eta}_{\mathrm{ch}}(k+l-i \mid k) & \\ +\sum_{i=1}^{n_{b}} b_{i} u(k+l-i+1), & \text { if } l \leq l_{\max } \\ \sum_{i=1}^{n_{a}} \alpha_{i} \hat{\eta}_{\mathrm{ch}}(k+l-i \mid k), & \text { else. }\end{cases}
$$

The coefficients $a_{i}, b_{i}$ of the ARX model and $\alpha_{i}$ of the AR model are estimated individually using the cost function given by (7), and the identified models were validated according to the variance of the prediction error, over a forecasting horizon $l$, given by (8).

Given an order $n_{a}=30$ for the AR model obtained in Section II-B, ARX models of different orders $n_{b}$ were trained

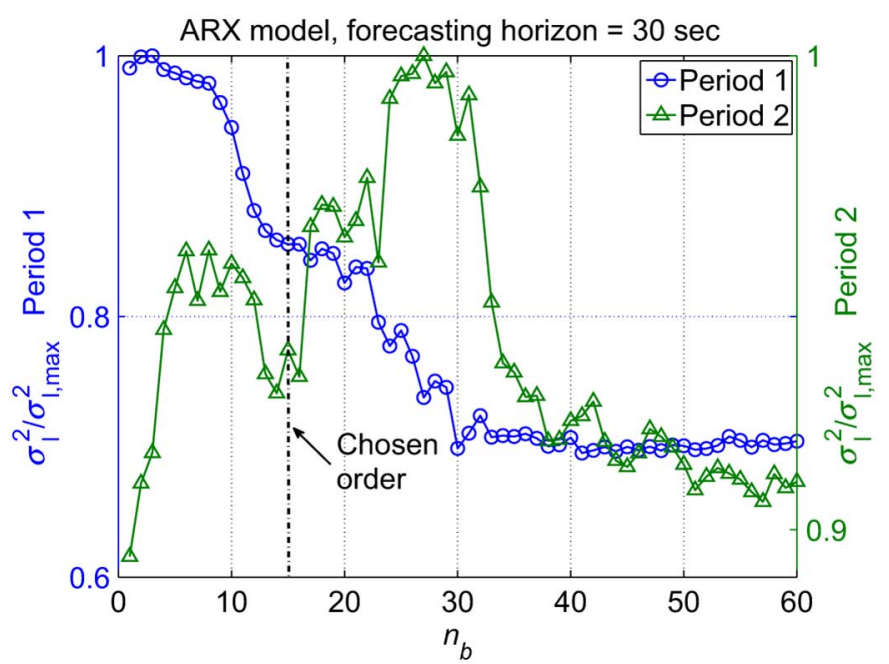

Fig. 6. Normalized prediction error variance for a forecasting horizon of $30 \mathrm{~s}$ as a function of the order $n_{b}$ with $n_{a}=30$ averaged across validation data sets for the first and second deployment periods.

and validated for both the deployment periods. The optimal order $n_{b}$ selected for the FIR model cannot be used directly for the ARX model, since that, in (11), the chamber wave elevation is not exclusively determined by the up-wave measurement, but it is also determined by its past values. In Fig. 6, the normalized variance of the prediction error, averaged across the validation data sets, is plotted against different orders $n_{b}$ for both the deployment periods. By inspection of Fig. 6, an order $n_{b}=15$ is selected as a trade-off point between the minima of the two curves. The order $n_{b}$, chosen for the ARX model, is different from the order $n_{b}$ of the FIR model, since the ARX models were trained and validated for a fixed choice of $n_{a}=30$.

\section{Neural Networks}

In this section, an NN based on multiple layers of perceptrons is presented in order to investigate the possibility of a nonlinear relationship between the free surface elevation up-wave of the WEC and the chamber wave elevation. Different architectures for the NN were considered, and the NN selected for the prediction of the chamber wave elevation is made of three layers of perceptrons. The first and second layers include three and five nonlinear neurons, respectively, while the output layer is made of one linear neuron. A linear neuron was selected for the output layer, in order not to limit the range of values assumed by the output of the NN. The inputs of the NN are composed of past values of the chamber wave elevation and free surface elevation up-wave of WEC. The structure of the NN is of the following form:

$$
\begin{gathered}
\eta_{\mathrm{ch}}(k)=\mathbf{N N}\left(\eta_{\mathrm{ch}}(k-1), \ldots, \eta_{\mathrm{ch}}\left(k-n_{a}\right),\right. \\
\left.u(k), \ldots, u\left(k-n_{b}+1\right)\right)
\end{gathered}
$$

with regression orders $n_{a}$ and $n_{b}$ equal to the orders selected for the linear ARX model, i.e., $n_{a}=30$ and $n_{b}=15$. The same orders selected for the linear ARX model were also adopted for the NN-ARX, in order to have a consistent comparison between the performance of the two models. The NN was trained using 


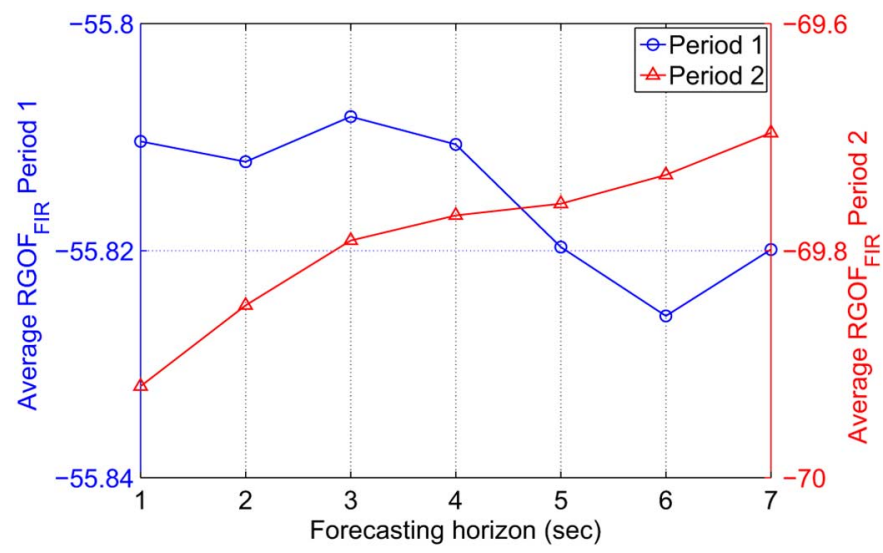

Fig. 7. RGOF between AR model $\left(n_{a}=30\right)$ and FIR model $\left(n_{b}=10\right)$ averaged across the validation data sets of the first and second deployment periods.

the Levenberg-Marquardt backpropagation algorithm [16] on a set of batch data, following which the network was used to compute the $l$-step ahead prediction of the chamber wave elevation.

\section{RESULTS}

In the following section, the forecasting of the water level in the chamber given by the FIR and ARX models is compared with the forecasting given by the AR model over a certain range of prediction horizons. The index used for the prediction accuracy for every forecasting horizon $l$ is the goodness of fit (GOF) index, which is defined as follows:

$$
\operatorname{GOF}(l)=\left(1-\frac{\sqrt{\sum_{k}(\eta(k+l)-\hat{\eta}(k+l \mid k))^{2}}}{\sqrt{\sum_{k} \eta(k)^{2}}}\right) \cdot 100 .
$$

In order to compare the performance between the AR model and the FIR or ARX model, the relative goodness of fit (RGOF) is introduced

$$
\begin{aligned}
\operatorname{RGOF}_{\mathrm{FIR}}(l) & =\operatorname{GOF}_{\mathrm{FIR}}(l)-\operatorname{GOF}_{\mathrm{AR}}(l) \\
\operatorname{RGOF}_{\mathrm{ARX}}(l) & =\operatorname{GOF}_{\mathrm{ARX}}(l)-\operatorname{GOF}_{\mathrm{AR}}(l) .
\end{aligned}
$$

The RGOF between an AR model of order $n_{a}=30$ and a FIR model of order $n_{b}=10$, averaged across the validation data sets, is plotted against the forecasting horizon for the first and second deployment period in Fig. 7. As shown in Fig. 7, the AR model is able to provide more accurate predictions of the wave elevation in the chamber for all forecasting horizons up to 7 s. In Fig. 8, the RGOF between an AR model of order $n_{a}=30$ and a ARX model of order $n_{a}=30$ and $n_{b}=15$, averaged across the validation data sets of the first deployment period, is plotted against the prediction horizon for different values of the cut-off frequency $\omega_{c}$. As the figure shows, the AR model performs better than the ARX model for prediction horizons that range from $10 \mathrm{~s}$ up to 22 or $27 \mathrm{~s}$, depending on $\omega_{c}$. The choice of the cut-off frequency $\omega_{c}$ depends on frequency components of the chamber wave elevation that need to be predicted. Since the resonance of the chamber is around $0.6 \mathrm{rad} / \mathrm{s}$,

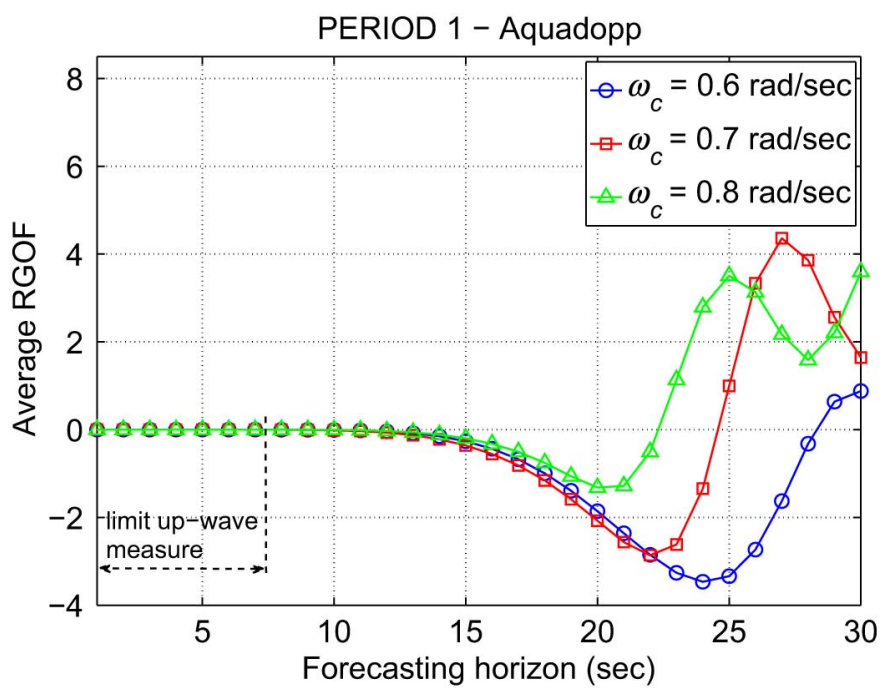

Fig. 8. RGOF between AR model $\left(n_{a}=30\right)$ and ARX model $\left(n_{a}=30\right.$, $\left.n_{b}=15\right)$ averaged across the validation data sets of the first deployment period.

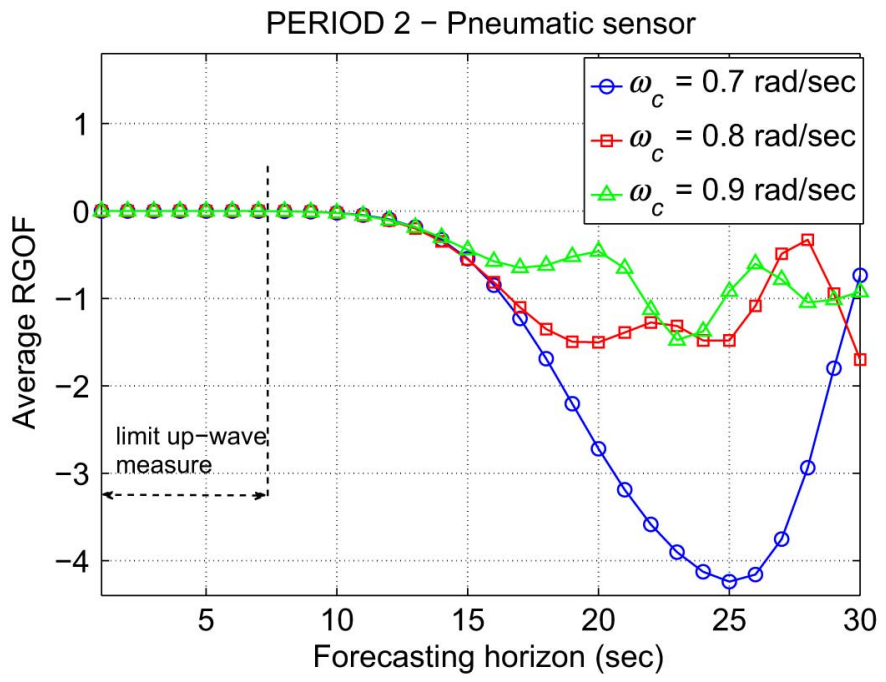

Fig. 9. RGOF between AR model $\left(n_{a}=30\right)$ and ARX model $\left(n_{a}=30\right.$, $\left.n_{b}=15\right)$ averaged across the validation data sets of the second deployment period.

a cut-off frequency $w_{c}=0.7 \mathrm{rad} / \mathrm{s}$ is selected for filtering the chamber and up-wave elevation. Note that the improvement in the accuracy of the prediction of the chamber wave elevation for long horizons obtained with the ARX model has to be evaluated respect to the prediction requirements in the control of the WEC. In fact, because of the noncausal transfer function involved in the realization of the real-time optimal control of the WEC, the future wave elevation may be needed until a time horizon less than $30 \mathrm{~s}$ [17]. Regarding second deployment period, in Fig. 9, the RGOF between an AR model of order $n_{a}=30$ and an ARX model of order $n_{a}=30$ and $n_{b}=15$, averaged across the validation data sets, is plotted against the prediction horizon for different values of the cut-off frequency $\omega_{c}$. As the figure shows, the ARX model provides less accurate predictions of the wave elevation inside the chamber than the AR model for every prediction horizon. 


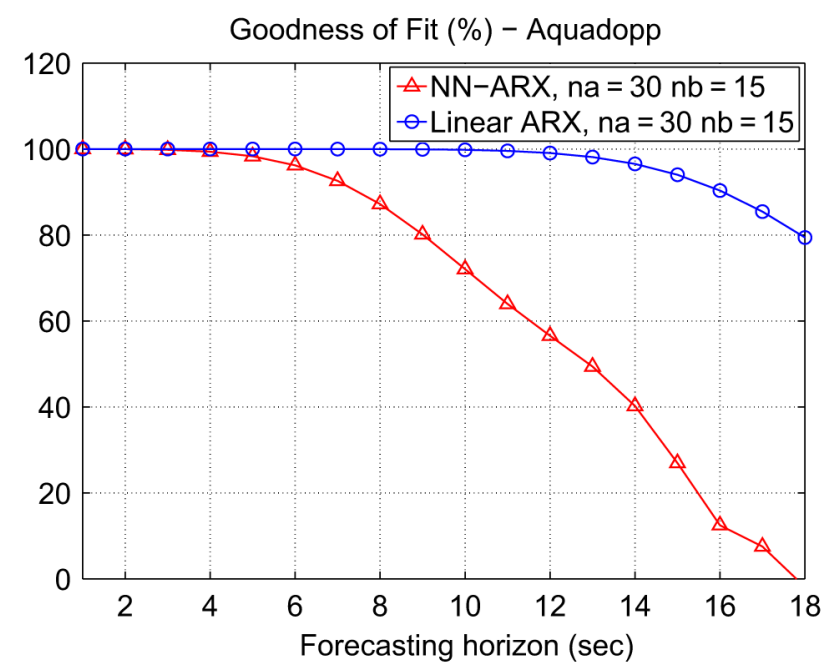

Fig. 10. GOF of linear ARX model $\left(n_{a}=30, n_{b}=15\right)$ and of NN-ARX model $\left(n_{a}=30, n_{b}=15\right)$ computed on validation data set of the first deployment period.

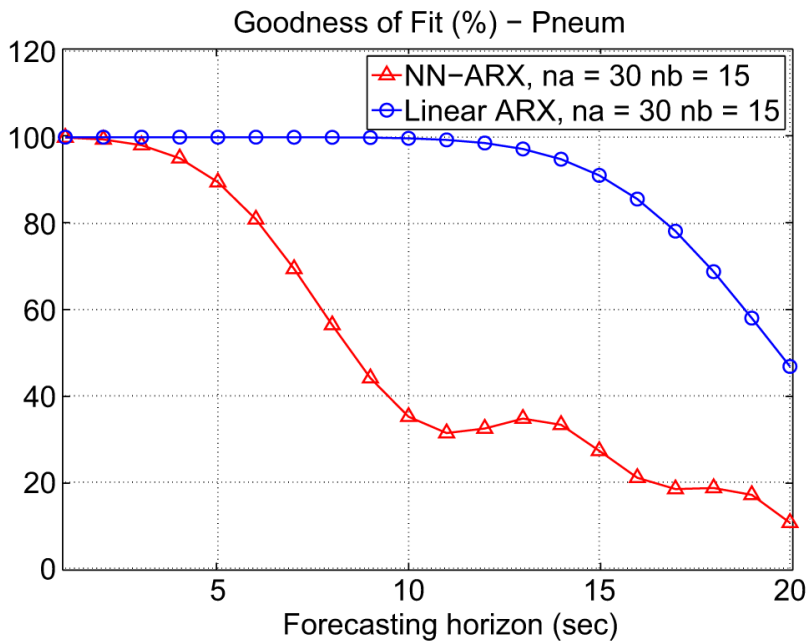

Fig. 11. GOF of linear ARX model $\left(n_{a}=30, n_{b}=15\right)$ and of NN-ARX model $\left(n_{a}=30, n_{b}=15\right)$ computed on validation data set of the second deployment period.

In Fig. 10, the GOF of the predictions of the chamber wave elevation given by the linear ARX model and NN-ARX model are compared for forecasting horizons up to a maximum of $20 \mathrm{~s}$. The two prediction models were trained and validated on the same batch of training and validation data from the first deployment period. Since the algorithm chosen for training the NN is highly dependent on the choice of the initial parameters for the network, different NN-ARX models were trained with different initial conditions, with the NN that returned the best GOF selected. In Fig. 11, the same comparison is done for the second deployment period. For both the deployment periods, the linear ARX model outperforms the NN-ARX model.

A comparison between predicted and actual chamber wave elevations for a forecasting horizon of $30 \mathrm{~s}$, for an ARX model of order $n_{a}=30$ and $n_{b}=15$, is made. The ARX model utilizes the free surface elevation up-wave of the WEC provided by the Aquadopp and pneumatic sensors, reported in Figs. 12

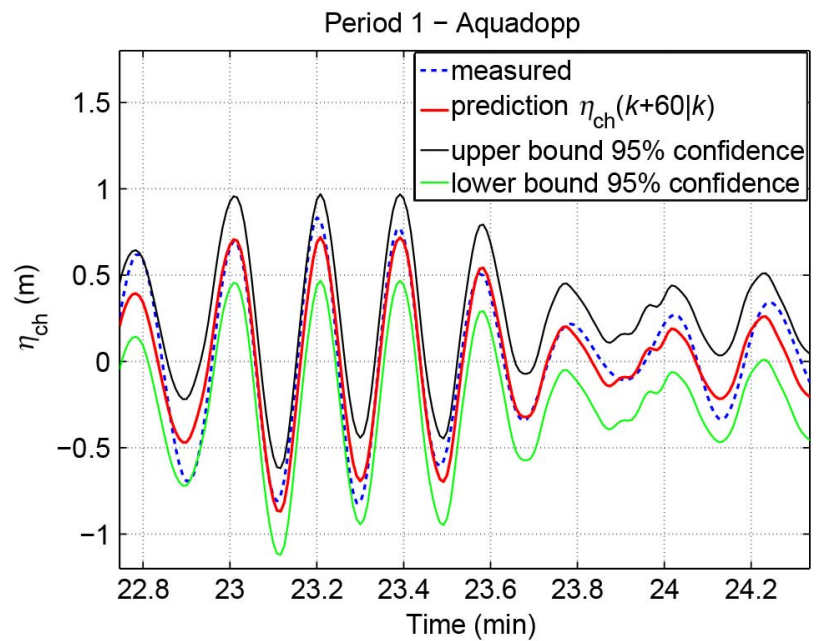

Fig. 12. Comparison between measured chamber wave elevation and prediction $\eta_{\mathrm{ch}}(k+60 \mid k)$ computed with ARX model of order $n_{a}=30$ and $n_{b}=$ 15 with data set of first deployment period, filtered with a cut-off frequency of $\omega_{c}=0.7 \mathrm{rad} / \mathrm{s}$.

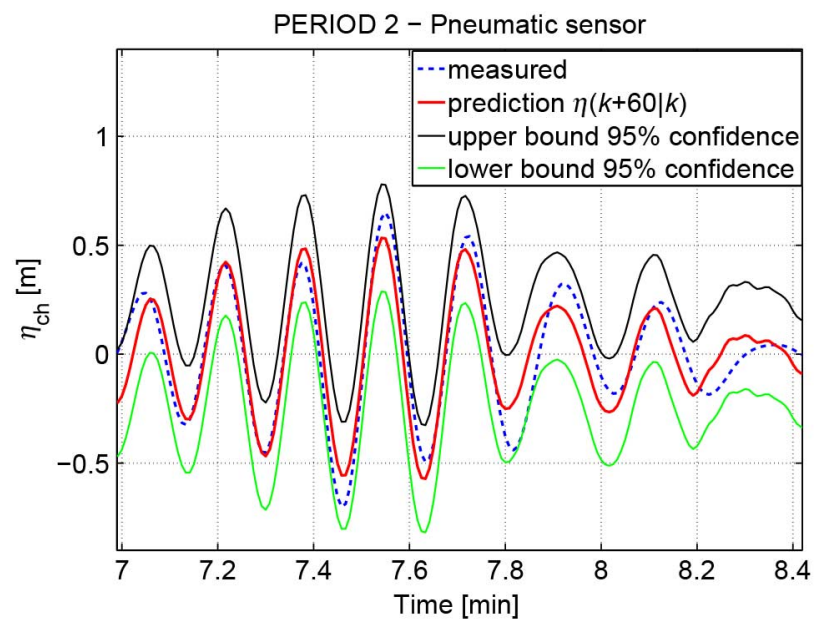

Fig. 13. Comparison between measured chamber wave elevation and prediction $\eta_{\mathrm{ch}}(k+60 \mid k)$ computed with ARX model of order $n_{a}=30$ and $n_{b}=15$ with data set of second deployment period, filtered with a cut-off frequency of $\omega_{c}=0.7 \mathrm{rad} / \mathrm{s}$.

and 13 , respectively. The prediction of the chamber wave elevation is plotted together with confidence intervals, which can be assumed to be a Gaussian process with zero mean and variance $\sigma_{l}^{2}$ given as follows [3]:

$$
\hat{e}(k+l \mid k)=\eta(k+l)-\hat{\eta}(k+l \mid k) \cong \mathcal{N}\left(0, \sigma_{l}^{2}\right) .
$$

\section{CONCLUSION}

This paper demonstrates that, for the case of the Pico OWC installation, there is little advantage in making an up-wave measurement, in terms of wave forecasting accuracy. This is useful information, allowing potential reductions in capital and maintenance costs. However, the generality of such a conclusion is uncertain. The Pico wave climate is reasonably stable, with a well-defined swell component. Furthermore, the forecasting problem for an OWC is straightforward, where the chamber 
water elevation is directly measurable, unlike the case of floating devices, where the free-surface elevation at the device location is impossible to measure, and the focus may need to change to excitation force. It should also be borne in mind that, for the current case study, the chamber was in an unloaded condition (relief valve open); one might expect some small change in the forecasting problem for a loaded chamber. Also, the paper examines the efficacy of using nonlinear forecasting models, showing no benefit. While the added complexity is a drawback in its own right, the fact that artificial NNs do not, by default, include the linear case suggest a preference for linear models, at least in the first instance. Finally, it is important to highlight that the noncausal control law computes the optimal velocity for maximum wave energy absorption, which depends on the future values of the wave elevation [17]. Prediction errors on the wave elevation cause the tracking of an incorrect velocity reference, and therefore a loss on the absorbed power occurs. In [18], a floating cylinder is considered, and the study has demonstrated that prediction errors on the wave elevation can result in losses of absorbed power from $8.5 \%$ to $40 \%$, depending on sea state considered.

\section{REFERENCES}

[1] U. A. Korde, "Control system applications in wave energy conversion," in Proc. OCEANS 2000 MTS/IEEE Conf. Exhib., Providence, RI, USA, 2000, vol. 3, pp. 1817-1824.

[2] J. Falnes, Ocean Waves and Oscillating Systems. Cambridge, U.K.: Cambridge Univ. Press, 2002.

[3] F. Fusco and J. V. Ringwood, "Short-term wave forecasting for real-time control of wave energy converters," IEEE Trans. Sustain. Energy, vol. 1, no. 2, pp. 99-106, Jul. 2010.

[4] J. Tedd and P. Frigaard, "Short term wave forecasting, using digital filters, for improved control of wave energy converters," in Proc. Int. Offshore Polar Eng. Conf., 2007, pp. 388-394.

[5] J. Dannenberg, K. Hessner, P. Naaijen, H. V. D. Boom, and K. Reichert, "The on board wave and motion estimator OWME," in Proc. Int. Offshore Polar Eng. Conf., 2010, pp. 424-431.

[6] M. R. Belmont, J. M. K. Horwood, R. W. F. Thurley, and J. Baker, "Shallow angle wave profiling Lidar," Atmos. Ocean. Technol., vol. 24, no. 6, pp. 1150-1156, 2007.

[7] J. R. Halliday, D. G. Dorrell, and A. R. Wood, "An application of the fast Fourier transform to the short-term prediction of sea wave behaviour," IEEE Trans. Renew. Energy, vol. 36, no. 6, pp. 1685-1692, Jun. 2011.

[8] M. Belmont, J. Horwood, R. Thurley, and J. Baker, "Filters for linear sea-wave prediction," Ocean Eng., vol. 33, no. 17-18, pp. 2332-2351, Dec. 2006

[9] V. Voronovich, B. Holmes, and G. P. Thomas, "A preliminary numerical and experimental study of wave prediction," in Proc. 6th Eur. Wave Tidal Energy Conf. (EWTEC), 2005, pp. 535-542.

[10] K. Monk, D. Conley, M. Lopes, and Q. Zou, "Pneumatic power regulation by wave forecasting and real-time relief valve control for an OWC,' in Proc. Eur. Wave Tidal Energy Conf. Series, Aalborg, Denmark, 2013 [CD-ROM].

[11] B. Fischer, P. Kracht, and S. Perez-Becker, "Online-algorithm using adaptive filters for short-term wave prediction and its implementation," in Proc. Int. Conf. Ocean Energy, 2012 [CD-ROM].

[12] M. P. Schoen, J. Hals, and T. Moan, "Wave prediction and robust control of heaving wave energy devices for irregular waves," IEEE Trans. Energy Convers., vol. 26, no. 2, pp. 627-638, Feb. 2011.

[13] A. Price and A. Wallace, "Non-linear methods for next wave estimation," in Proc. 7th Eur. Wave Tidal Energy Conf., 2007 [CD-ROM].

[14] U. A. Korde, "Up-wave surface elevation for smooth hydrodynamic control of wave energy conversion in irregular waves," in Proc. MTS/IEEE OCEANS'13, San Diego, CA, USA, 2013 [CD-ROM].

[15] D. S. Shook, C. Mohtadi, and S. Shah, "Identification for long-range predictive control," in Proc. Inst. Elect. Eng. D Control Theory Appl., 1991 vol. 138, no. 1, pp. 75-84.
[16] H. M. T. and M. Menhaj, "Training feed-forward networks with the Marquardt algorithm," IEEE Trans. Neural Netw., vol. 5, no. 6, pp. 989993, Aug. 1994

[17] F. Fusco and J. V. Ringwood, "A study on the prediction requirements in real-time control of wave energy converters," IEEE Trans. Sustain. Energy, vol. 3, no. 1, pp. 176-184, Dec. 2012.

[18] F. Fusco and J. V. Ringwood, "A model for the sensitivity of non-causal control of wave energy converters to wave excitation force prediction errors," in Proc. 9th Eur. Wave Tidal Energy Conf. (EWTEC), 2011 [CD-ROM].

Francesco Paparella received the Laurea Magistrale in automation engineering from the Politecnico di Milano, Milan, Italy, in 2012. Since October 2013, he is pursuing the Ph.D. degree at the Center for Ocean Energy Research, National University of Ireland Maynooth (NUIM), Maynooth, Ireland.

During his thesis, he worked on the load sharing optimization of compression stations at the ABB Corporate Research Center in Baden, Zurich, Switzerland. From January 2013 to September 2013, he worked with RSE S.p.a., Milan, Italy, as a Researcher for the development of electrical systems.

Kieran Monk received the degree in physics from Cardiff University, Cardiff, U.K., and the Master's degree in physical oceanography from Bangor University, Bangor, U.K. He is pursuing the Ph.D. degree at the School of Marine Science and Engineering, Plymouth University, Plymouth, U.K.

He is a Resident Engineer of the Pico OWC project for the company WAVEC, Lisbon, Portugal. In parallel to the Ph.D., since 2011 he has also been the Resident Engineer and Scientific Officer with the Pico OWC in the Azores and is jointly responsible for all aspects of operating, maintaining, and enhancing the machine, as well as the data management and research outputs.

Victor Winands is currently pursuing the Ph.D. degree in problems of wave engineering at Aalborg University, Aalborg, Denmark.

$\mathrm{He}$ is a Dipl. Engineer from Berlin, Germany, working with Duespanwave-engineering, Berlin, Germany. He worked on rebuilding, changing, and repairing the Pico wave energy plant until it reached permanent remote controlled operation in 2010. He is responsible for the Pico plant and all changes on it since 2008 .

M. F. P. Lopes received the Ph.D. degree in mechanical energy from the Instituto Superior Tcnico, Lisbon, Portugal, in 2011, on tank testing methods for wave energy converters.

He is the Head of the Technology Monitoring Team, WAVEC Offshore Renewables, Lisbon, Portugal, since 2011. Originally, he was a Construction Engineer. His research interests include multiple fluid mechanics and energy applications including wave-structure interaction, wave and offshore wind energy, and wind actions on buildings.

Daniel Conley received the Ph.D. degree in oceanography from the University of California at San Diego.

$\mathrm{He}$ is an Associate Professor (Reader) with the School of Marine Sciences and Engineering, Plymouth University, Plymouth, U.K. Prior to joining the University of Plymouth with a PRIMaRE appointment, he was an Assistant Professor with the State University of New York, Stony Brook, NY, USA, for 7 years and a Research Scientist with the NATO Undersea Research Centre, La Spezia, Italy, for a further 7. Currently, he is a Research Group Leader with the Coastal Processes Research Group (http://www.coastalprocesses.org/) as well as Pathway Leader with the UoP Marine Renewable Energy M.Sc. program. His research interests include resource assessment and impact monitoring for marine renewable energy, sediment transport, waves, turbulence, estuarine circulation, and coastal monitoring.

John V. Ringwood received the Diploma degree in electrical engineering from Dublin Institute of Technology, Dublin, Ireland, and the Ph.D. degree in control systems from Strathclyde University, Glasgow, U.K., in 1981 and 1985, respectively.

Currently, he is a Professor of Electronic Engineering with the National University of Ireland (NUI), Maynooth, Ireland. From 2000 to 2005, he was the Head of the Electronic Engineering Department, NUI, developing the department from a greenfield site. He currently directs the Centre for Ocean Energy Research, NUI. His research interests include time-series modeling, wave energy, control of plasma processes, and biomedical engineering.

Dr. Ringwood is a Chartered Engineer and a Fellow of the Institution of Engineers of Ireland. 